\title{
7. Machinising humans and humanising machines: Emotional relationships mediated by technology and material experience
}

\author{
Caroline Yan Zheng \\ Royal College of Art \\ Yan.zheng@network.rca.ac.uk
}

(Author Accepted Manuscript, submitted 26 April 2016)

\begin{abstract}
With the advent of affective computing and physical computing, technological artefacts are increasingly mediating human emotional relations, and becoming social entities themselves. These technologies on one hand prompt a critical reflection on human-machine relations, and on the other hand offer a fertile ground for imagining new dynamics of emotional relations mediated by technology and materiality. This chapter describes design research drawing on theories of technology, materiality and making. Carried out through fashion and experience design, my practice amplifies processes of mediation. By creating material playgrounds for technological and human agency, the experiments described here aim to generate knowledge about the emotional self, critical reflection on human-machine relationships, and new imagined emotional relations resulting from the hybridity of humans and technology.
\end{abstract}

\section{Background}

Although it has been recognised that technology mediates human existence in the world, as well as how the world is presented to humans (Ihde, 1990), emotion is considered core to what makes us human, and in general we trust humans over technologies in emotional matters. What happens when this space is mediated by technological artefacts, and machines 
are equipped with a degree of emotional intelligence, thereby gaining an identity as social creatures?

Information technologies addressed to emotions have so far been constrained mainly to sensing technologies and analytics. Picard coined the term 'affective computing' to refer to 'computing that relates to, arises from, or influences emotions'(1995: 1). On one hand, the aim is new self-understanding and communication of human emotions; on the other hand it aims at designing computers with better 'emotional intelligence' that is, 'the ability to sense and recognise expressions of human emotion' which helps 'machines choose more helpful and less aggravating behaviour'(Picard, 2007: 396). In other words, technology is simultaneously machinising humans and humanising machines. With the growth of affective computing technologies, we see the phenomena of technological artefacts intervening in human emotions.

In the intrapersonal space, wearable gadgets such as Empatica and various mobile apps monitor and share wearers' emotional status. In the interpersonal space, there is research outcome for example VibroGlove (Krishna et al., 2010) which detects other people's emotional states using facial recognition and sends vibration feedback to inform a wearer who is visually impaired; and SWARM which can detect group emotional states and inform its wearer 'if the mood in the room she is about to enter is tense or relaxed' (William et al., 2015). Mobile apps such as Headspace aims to guide users towards 'spacious, focused, relaxed and content' states of mind through meditation, and claim to connect a community of more than two million users across the world (Barol, 2015). Art projects such as AWE Goosebumps (Neidlinger et al., 2015) attempt to translate our innermost feelings to the external world. Software such as Emotient can sense people's emotions distantly through 
facial recognition, while technologies such as sentiment analysis mine data to indicate largescale public emotional states (Liu, 2015).

Simulated emotional intelligence has been built into so-called ambience intelligence systems (Lugmayr et al, 2009) and sociable robots. Both aim to be able to adjust their behaviour to adapt to human emotional cues. Sociable robots are developed to communicate with humans emotionally and to become companions and carers. Their lifelike qualities stem from their anthropomorphic character (Złotowski et al., 2015). These animated machines aim to perceive and react to humans, seeming to demand to be treated as new social entities. Some researchers already suggest a rethinking and redefining of emotion, and argue that robots can have emotions similar to humans, even if not exactly equivalent to those of humans, (e.g. (Hamilton 2014).

Thus every aspect of human emotion has been stirred up by technologies and mediating artefacts. There are confusions and concerns, but also imagination of new dynamics of human-machine relations in this intimate space. However, the plural dimensions of emerging relations are under-explored. Wearable, affective artefacts are mainly developed and presented as products or services, with prescribed machine behaviour already programmed in. What we are offered is a product-user relation, accompanied with a given assumption that these technological artefacts are to make our lives happier, and we are seemingly expected to accept and adapt to them. Given the multi-dimensional influence of technologies and the fact that there is not even agreement among emotion theorists on 'what emotions are and how we can represent them' (Lopatovska and Arapakis, 2011), we risk missing many opportunities if the knowledge and relations in this new emotional space are not explored in multiple dimensions. Rather taking as given a one-dimensional human-machine relation, my research 
accepts this emotional space as 'a place of open-ended emergence and becoming' (Pickering and Guzik, 2008: ix), in which 'both the human and the nonhuman are recognised as openendedly becoming, taking on emergent forms in a intrinsically temporal 'dance of agency', (Pickering and Guzik, 2008: 1).

\section{Theoretical grounding}

\section{Human-machine relationships}

The human-machine relationship for me has two layers. On one layer is the extent to which humans and machines are different from or similar to each other. As humans tend to build tools inspired by or resembling our own physical bodies and their functions, machines take on a human character to varying degrees, and we recognise ourselves in some machines. For affective computing to 'machinise humans' is an activity of collecting and analysing human data and recognising behavioural patterns over time. These behavioural patterns are then modelled and installed on machines as artificial intelligence. To perfect this human-machine relationship, especially on an individual level, motivates us to gain knowledge about our emotion selves.

The other layer of human-machine relationships I consider is how we live with machines as mediators of our lives. Viewing technological artefacts largely as products of human users, philosophers of technology who deal in post-phenomenological approaches see limitations in the modernist view that separates human subjects and technology objects (e.g. Latour, 1993; Ihde, 1990; Verbeek, 2015). Post-phenomenology brings mediation to the forefront of human-technology relations. According to Peter-Paul Verbeek, 'humans and technologies 
should not be seen as two 'poles' between which there is an interaction', but in an intertwined relation of mutually shaping each other. The relation between humans and technologies is 'part of a larger relation, between human beings and their world, in which technologies play a mediating role' (Verbeek, 2015: 28).

Andrew Pickering (2008) criticises modernity as marked by a mode of 'enframing' in which 'we humans seek to step outside nature, dominating and controlling it, challenging it forth as ‘ 'standing reserve' for circuits of production and consumption' (Pickering, 2008: 5). He thus sees asymmetry in the relations in which a human agent outweighs that of a non-human agent. In the case of human emotions mediated by technology, I see as well an asymmetry, but in reverse, as the dominance is on the side of technological agents over human subjectivity. The emotional cues measurable by technological agents are valued and amplified, while as yet unmeasurable aspects of human subjectivity are under-treated.

If the promise of affective computing is to offer tools 'to know about ourselves better and communicate better', this self-empowerment is under-explored while technologists, scientists and designers are busy pushing forward the other end - making smarter machines. Technologies are employed to push inch by inch the quantifiable boundaries of human emotion; and scientists, including physiologists, neurologists and psychologists, have joined forces with engineers to teach computers to look out for these measurable indicators of human emotion. It is the human agent, then, that is treated more like a 'standing reserve', or a treasure pot for data extraction, in order to develop smarter machines, so as to serve the production and consumption of technologies. 
All this is promoted as satisfying human needs and complimenting human deficiencies, under the logic that we will empower ourselves if we have powerful machines. But how might technological artefacts be used to empower individuals in learning about themselves in alternative ways?

Different disciplines regard differently the relation between humans and technological artefacts. In sociable robotics, researchers make clear that when speaking of robot emotions, they mean a strictly functional sense. The question of whether robots could really have and feel human emotions is irrelevant for their purposes (Breazeal and Brooks 2005). Sherry Turkle raises a deep concern over what she calls the 'robotic moment' (Turkle, 2011: 40), wherein we delegate important human relationships to sociable robots, while 'we are losing the raw, human part of being with each other' (Turkle quoted in De Lange, 2013).

For Verbeek, approaching technological artefacts as mere instruments, as extensions of human beings, or as opposed to human-human relationships, falls back to the modernistic split between subject and object. This separation, he says, fails to grasp the intertwined relations of humans and technologies (Verbeek, 2015:29). He thus proposes thinking of these relations in terms of 'hybrids' in which technologies and human beings mutually shape each other; there is no pre-given subject and object (Verbeek, 2015). The influencer and the influenced and various other relations take shape through mediation and a process of mutual constitution.

My research thus refuses the taken-as-given approach and the 'enframed' description that technology is there to make our emotional lives happier or to compliment our sensorial deficiencies. But nor do I take the opposite stance, criticising technologies for alienating 
human-human relations. I see the disrupted emotional space as a place of open-ended emergence and becoming, as Pickering (2013) terms it, in which technology and humans are intertwined in hybridity, as a starting point.

Coming from a Chinese background, my stance also reflects the influence of Chinese philosophy, which emphasises not absolutes but relativity. The search for wisdom is to recognise emerging trends and seek a dynamic balance in constant change. The dynamic balance is in both human-human relations as well as between human and nonhuman agents.

\section{Hybridity with materiality}

Theories about making identify the mediating power of materiality, and propose that we reconsider the human-matter relationship to actively explore new dynamics through critical reflection during the process of making. 'Whether into a technically advanced or technically primitive society, the world is produced... we are ineluctably constituted in the artificial... What we make alters, in small or large measure, the world around us and our being-in-the world' (Rosenberg, 2013:1).

It used to be that technologies were focused on mediating virtual experiences through the screen, and the making and computing communities were considered extremely different. Now with physical computing (O'Sullivan and Igoe, 2004), technologies are able to exert their mediating influence through the materiality of technological artefacts. Objects become alive, responsive and even adaptive, and technological mediation becomes physical and visceral. The boundaries between body, space, physical and digital have merged. Here, technology and materiality become one; coding and making interweave; the mediating power of computation couples with that from the aesthetics and sensual qualities of artefacts. 
However, this hybridity also adds layers of complexity to already sophisticated affective computing technologies.

In this context, a deductive approach utilising controlled inputs to develop affective artefacts reduces complexity, while a linear approach in which technology is developed separately before passing on for the design of material interfaces also misses the potential of this hybridity. My research on the hybridity of technology, materiality and human agency aims to gain insight through mutual influence.

With a hybrid approach to explore new dynamics of emotional relations mediated by technology, it is the process, rather than the end product, that opens doors for new meanings and relations. Drawing upon the work of Deleuze and Guattari, Tim Ingold proposes 'an ontology that assigns primacy to the processes of formation as against their final products, and to the flows and transformations of materials as against states of matter' (2010: 92). In designing affective artefacts, some designers pre-suppose a scenario of specific application or identify a need or problem, then develop technology to address it. The approach in my research does allow the process of mutual constitution of human and mediated materiality from the very start, articulating the shaping of meaning and relations throughout the process. Mine is also a process of cross-disciplinary dialogue - only through dissolving conventional boundaries between design, social science, craft, engineering, science and technology, can a new landscape of relations emerge.

Verbeek recognizes that 'the field of interaction design is a rich source of inspiration for philosophy of technology, as the place where new types of human-technology relations emerge' (Verbeek, 2015: 31). In the case of digital-physical objects, Kevin Walker and John 
Fass (2015) ask how we can enhance, augment, represent or disrupt this experience, and disentangle the observable relationships to evoke a reflection on the process of being relational. In such a space of emergence and becoming, Pickering advocates a 'dance of agency' between people and things, in 'performative fashion' for exploring the unknown, as opposed to traditional causal interpretations of actions, instead seeing 'knowledge as growing out of them' (Pickering, 2013: 78-9).

\section{Methodology}

Thus, grounded in design and drawing on theories of technology, materiality and making, my research is carried out through fashion and experience design. The experimental practice described below is aimed at amplifying our understanding of human emotions as mediated by technology. By creating playgrounds for both technological and human agency - especially the agency of human subjectivity - my practice explores knowledge about the emotional self generated from within, in order to facilitate critical reflection on human-machine relationships, and to imagine new dynamics of emotional relations mediated by technology and material experience. My practical working methods, described below, involve making the intangible tangible, avoiding pre-assigned meanings, taking a heuristic approach, and treating humans, technologies and materials as performing in tandem.

\section{Making the intangible tangible}

Revealing 'the continuity between humans and nonhumans', as Rosenberger and Verbeek state, 'makes it possible to understand how nonhuman entities do not only play a role in the material world, but in the social world as well' (Rosenberger and Verbeek, 2015: 19). Walker and Fass (2015) describe a methodology termed 'de-computation' - a reverse design 
process, applying computational thinking to real-world phenomena in order to reveal and critique the role of technology and to uncover previously unseen design directions. Using this methodology, I follow two parallel lines: On one hand I employ technology to make tangible the invisible forces that influence human emotions, in order to explore how technology informs our knowledge of the self that otherwise might not be attained. On the other hand, I make tangible how technology disrupts our conventional emotion relations and mechanisms, revealing new reflections and meanings.

\section{Avoiding pre-assigned meanings and keeping the feedback loop open}

A common approach in artistic projects of emotional data manifestation is that sensors collect data indicating emotional status from biometric sensors, manifested initially as a set of numbers. These data need to be mapped onto another register such as colour or shape. But the language of these registers does not accord seamlessly with the physiological data. The designer has therefore to make decisions in categorising emotion data, sometimes arbitrarily assigning meaning to one or another category. If affective artefacts have limited behaviour, e.g. they have only five colours available, the designer needs to prescribe particular behaviours to them. In general, in all the practical projects described below, I try to avoid assigning meaning and prescribing particular behaviours to artefacts, instead directly mapping the sensed data without manipulation or appropriation, whenever possible. This is to allow a maximum of free, subjective interpretation from the human participants. As Wilkinson-Webe et al. describe:

Sensor readings are not "emotion" per se, in all its human richness. Although sensors do abstract numbers from the phenomena they sense, these phenomena are often not identical to the real phenomena of interest. Yet, by virtue of the 'reading' being 
abstracted from the measuring event, the door has been opened for a slippage from a modest heuristic to a more ambitious hermeneutic. The threads that can otherwise situate an "emotion indication" as partial knowledge can be cut by the design choices made.

(2016: 117)

\section{Heuristic approach}

My experimental practice take a heuristic approach - in that the designer devises an initial interaction that facilitates both human and technological agencies to perform in tandem, so as to facilitate the generation of meaning through a process of hybridity. Meanings inspired from this heuristic interaction will then be selectively developed into new relational material for new interactions. This results in cross-modal outcomes, and performative, embodied interactions - specifically hybrid objects and experiences, relational artefacts and performative installations using smart materials and textiles. In all my projects I thus try to avoid indicating particular forms or scenarios, so as to allow space for subjective interpretation and imagination from the participants.

\section{Technology, humans and materials perform in tandem}

Artefacts on their own are incomplete. They are the heuristic props to invite interplay from humans. In this manner, to facilitate the 'dance' (Pickering, 2013: 78) of the agency of technology, in tandem with the richness of human subjectivity in its ambiguity, as a way of exploring the unknown I emphasise individual experience and the generation of knowledge from 'within'. 
For an individual, knowledge of the self and the role of technology is often taken as given (Turkle, 2011: 123) or as top-down from experts or scientists. However, due to the complex and vague nature of human subjectivity, and in particular the emotional self, science has only started to understand this relation with the help of technology. Now individuals can use technological tools to gain knowledge of their emotion selves. As with hybridity, what certain technological artefacts mean during the process of interplay vary with different people and at different times, thus are highly context-dependent and materially situated (Rosenberger and Verbeek, 2015: 28). The individual subjective experience and the knowledge generated from within should be valid as knowledge of the emotional self, potentially more valuable than knowledge acquired from outside.

\section{Experimental practice}

The projects described in this section are first few steps of a long journey. Though preliminary, they represent fresh new attempts at exploring a design research practice consisting of devising technological artefacts and material experiences designed to facilitate critical reflection, the generation of new meanings, and novel dynamics of emotional relations in the mediation of technology.

\section{Project \# 1: Tangible Emotions}

By connecting physiological data with kinetic installations in space, Tangible Emotions \#1 explores a novel employment of biometric data on emotions as a resource for communication and live performance. It also investigates a new dynamic in the performative interaction of technological and human agencies. In previous wearable, affective artefacts and data visualisations, data from sensors is normally displayed or transcribed in a linear way. This installation instead generates open feedback loops. 
Some pebble stones, lying on a stool among white sand, are embedded with pulse sensors.

Their size and oval shape invites holding in the hand, and subtle blinking lights invite interaction. When a participant picks up a pebble and holds it in the hand, a red light starts to blink, indicating the participant's pulse rate. A fabric installation in the space is then triggered, spinning at a speed according to the sensed pulse rate. Participants can thus manipulate the fabric's movement by manipulate their heart rate, for example by meditating or running around. Three people can do the experiment at the same time, making the installation a collective choreography (See Figure 1).

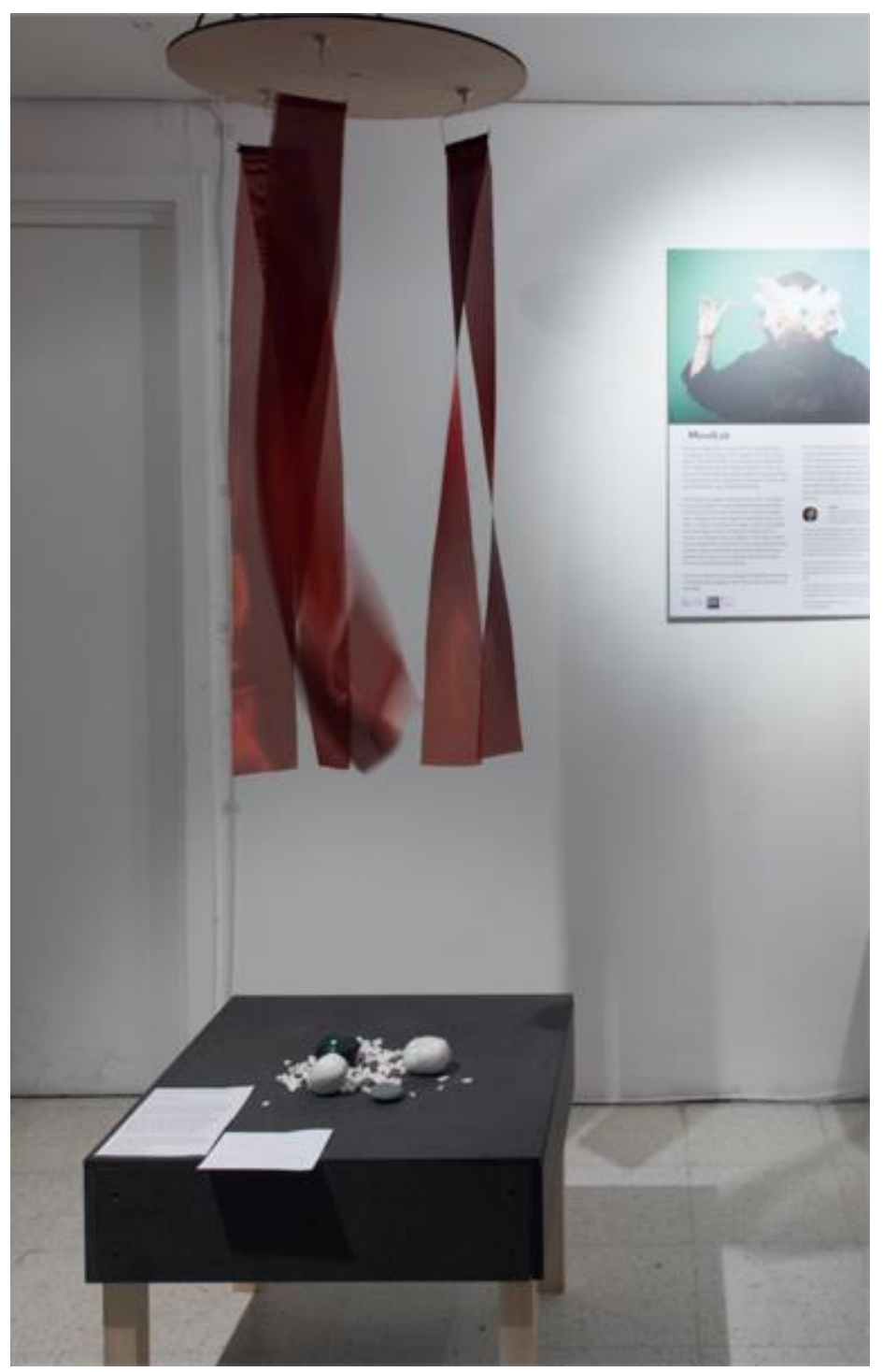

Figure 1. Tangible Emotions installation at RCA Work in Progress show, January 2016, Caroline Yan Zheng 
Heart rate is also an indicator of stress or anxiety. In this installation, stress is transcribed to a different modality as a source of aesthetic performance. My intention is to 'de-automate habitual perception and facilitate a "seeing freshly"'(Davis quoted in Hansen, 2006: 111). Feedback in my installations is not unidirectional; the kinetic movement of the textiles in turn influences the emotions of the participants. And unlike most wearable artefacts which utilise individual human-machine interaction, here when more than one person participates, it suddenly becomes a human-human interaction, mediated by technological artefacts.

I found that the size, colour, shape, and kinetic patterns of the materials evoke different emotional responses from participants. For example, when the textile is scaled up to three times the bodily size of participants, they express feelings of empowerment, and when the material changed to skin-coloured soft silicone, participants express fear. This suggests links between the language of materiality of such kinetic and responsive interfaces and their psychological impact.

One participant commented that he felt that he is 'part of the installation' and the installation is part of him, as it was the same heart that powered both his body and animated the visible movement of the fabric. Might such a connection between biophysical data and kinetic physicality constitute an embodied tangible notion of an 'extended-self' or out-of-body experience? Another participant offered that she would like to see such an installation much bigger, for example in a train station, allowing many people to interact, perhaps via an app on their phones. She imagined such a public installation might give people a sense of wonder or a serendipity of romance while waiting. 
These topics came out through the interplay of human subjectivity, material and technological agents. This experiment to connect biometric emotion data to a kinetic installation in space will continue as it informs new dynamics.

\section{Project \#2: Tangible Emotions 2/Digital Impressionism}

Tangible Emotions 2 explores how technology might help us gain knowledge about the emotion self and its invisible relations with the world. It explores this through embodied, metaphorical technological artefacts. The project amplifies the invisible force of human emotional sounds in the environment that act upon embodied artefacts.

The project made use of software developed through a project called Digital Impressionism, run by Dr Laura Ferrarello in the Information Experience Design (IED) program at the Royal College of Art in London. Digital Impressionism is a platform for exploring new material possibilities of digital and physical materials, by modelling not only objects and environments but invisible forces that act upon them (Ferrarello \& Walker, 2016). This platform developed software to turn physical data into a 3D pointcloud; each of the points in the pointcloud then reacts to real-time audio input, which manipulates the $3 \mathrm{D}$ forms on the screen, resulting in new hybrid forms. These new forms can then be 3D printed and thus brought back into the real world as physical artefacts.

I specifically investigated how different human emotional sounds could affect the shape and surface of an object. I used a Microsoft Kinect depth camera to capture the shape of a balloon, which acted as a simplified representation of the body, into a pointcloud on the screen. The captured form was then exposed to different human emotional sounds - singing, 
humming, laughing, quarrelling and shouting, to observe how the various frequencies of each sound affected the form.

A random round object with smooth surface was used in the first round of heuristic experiments. The interaction was inspired by bodily artefacts in various cultures, including in Chinese culture, wherein important paraphernalia are treated as an embodiment of the self. Here, 3D printed jewellery was in turn an embodiment of the body, and of personal emotions. This project thus offers a new imagination of dynamic fashion accessories as part of the body, which remind us that we are constantly in the process of being shaped by both technology and emotions. Technology assists in revealing the intangible forces that act upon humans and generates knowledge of our selves in relation with the environment.

\section{Project \#3: Coach_Exoskeleton}

Using a similar method as in the previous project, this project also uses technology to reveal the process of technological mediation. By disrupting conventional emotion relations through the mediation of technological artefacts, it aims to facilitate better understanding of our own emotions, to generate knowledge from within.

Some (e.g. Turkle, 2011) question whether a robot can simply performs human bodily behaviours without having any feelings. This raises the further question whether the so called 'emotional intelligence' of machines is at all equivalent to human emotions, as well as a question for ourselves that can we cause ourselves to 'feel' emotionally, simply by enacting certain gestures? In other words, is emotion generated from the inside out, or the outside in? Scientific research (e.g., Myers 2005:513) suggests for example that the shape of a smile or frown releases chemicals which affect our emotional status. 
The artist Pia Lindman, in her research on grief, collected images of mourners from the New York Times, extracting bodily gestures from the pictures in drawings, then re-enacted the gestures herself. 'In her enactments of grief, Lindman felt her body produce a state of mind' (Turkle, 2011: 137). Lindman found that:

Grief is always expressed in a set of structured patterns, programmed, she thinks, by biology and culture. So we, like the robots, have programs beneath our expression of feelings. We are constrained by mechanisms, even in our most emotional moments. And if our emotions are mediated by such programming, asks Lindman, how different are our emotions from those of a machine?

(Turkle, 2011: 137)

How might we devise an interactive experience to enable people to find their own subjective experiences in this debate? Drawing from this, together with IED Visiting Lecturer Dr Johanna Bolton, we created a set of performative artefacts worn on the body. Exoskeleton joints are connected by programmable materials to generate movements. When the wearer is situated in certain emotional states, the exoskeleton gently nudges the wearer's body into an opposing gesture, to generate a disruptive emotion.

In one experiment for example, a participant is asked to 'feel proud' through his bodily gestures and by giving a speech. While stretching his body and neck, the lobster shell-like exoskeleton worn on the back of the wearer 'decides' to contract. This 'jacket' thus nudges the participant into a more humble gesture. Could one continue to perform the emotion of pride while interacting with such a mediating technological artefact? In this case, a 
participant reported that he suddenly felt rather 'small' than proud, providing tentative evidence that performing an emotion could then generate that emotion, but could conversely be impacted by technological artefacts and the emotional 'intelligence' they might carry. Might this help us to learn more about our emotional selves? Our approach in the IED programme is that artistic research of this sort is equally valid to scientific research for addressing such questions.

\section{Discussion}

Technologies about the body used to be equivalent to fashion - in its Latin root -factio- refers to the act of making (Bruno, 2002:123). With digital technologies, fashion can play a more direct role in mediating emotional relations by curating the body's cultural existence (Ryan 2014) through artefacts. This is an exciting space for fashion to extend its body-bound position into space, while still designing relational experiences that evoke reflection. In all three projects described in this chapter, the fashion practice, both in static and kinetic form, has contributed in aesthetic terms and in soliciting participants' engagement in interactive activities.

Compared with text-based discussions commonly employed in social science, a physical, material experience is a powerful medium to facilitate reflection, as it allows a full-body sensorial exchange of information. Although the physical has today been largely reduced to commodity and product, and our relationship with materiality has been reduced to userproduct or user-service models, in ancient China, the physical metaphor is the main embodiment of philosophical discussion and reflection. It permeates almost all major philosophical texts as well as poems. The projects described in this chapter are part of a 
design practice aiming to draw inspiration from such alternative ways of being in our relations with materiality.

In exploring new dynamics in emotional spaces mediated by technological artefacts and materiality, and in aiming to generate knowledge about the emotional self from within, my theoretical stance and the chosen methodology are aimed at soliciting attention and engagement from human agents, especially in liberating their subjective imagination of technological relations with our emotional selves. However, the outcomes are manifold and hybrid, both qualitative and qualitative, in various modalities and directions. Subsequent rounds of heuristic development of the three projects has already taken very different directions. The open-ended approach expects that implications generated from the process of mutual constitution will further take the research into different directions. It is thus a challenge to process the information generated from heuristic steps to evolve the research, while remaining focused on embodied, mediated emotion. My next steps thus involve a critical evaluation of the limitations and advantages of this practice.

Overall, my research works toward developing a methodology for design practice of devising technology-mediated material experiences to facilitate the interplay of humans, technology and materiality, in order to generate both empirical and subjective knowledge about our emotion selves and human-technology relations. Such choreography stages a ground for critical, cross-disciplinary dialogue among social science, the material artefact making, experience design, and technology. 


\section{References}

Barol, Bill (2015) The Monk And The Mad Man Making Mindfulness For The Masses, January 28. http://www.fastcompany.com/3041402/body-week/the-monk-and-the-mad-manmaking-mindfulness-for-the-masses (accessed Mar 30, 2016).

Breazeal, Cynthia, and Brooks, Rodney (2005) 'Robot emotion: A functional perspective', pp. 271-310 in Jean-Marc Fellous and Michael A. Arbib (eds) Who needs Emotion?. New York: Oxford University Press.

Bruno, Giuliana (2002) Atlas of Emotion: Journeys in Art, Architecture, and Film. New York: Verso.

Hansen, Mark B. N. 2012. Bodies in Code: Interfaces with Digital Media. New York and London: Routledge.

De Lange, Catherine (2013) 'Sherry Turkle: “We're Losing the Raw, Human Part of Being with Each Other"' The Guardian. May 5.

https://www.theguardian.com/science/2013/may/05/rational-heroes-sherry-turkle-mit (accessed Mar 30, 2016).

Delancey, Craig (2009) 'Foreword' in Jordi Vallverdy and David Casacuberta (eds)

Handbook of Research on Synthetic Emotions and Sociable Robotics: New Applications in Affective Computing and Artificial Intelligence, pp. xxi-xxii. Hershey and London:

Information Science Reference

Ferrarello, Laura and Walker, Kevin (2016) 'Shaping the form of sound through hybrid materiality'. Proceedings of SIGGRAPH (under review).

Hamilton, Cameron (2014) 'On the Possibility of Robots Having Emotions', Department of Philosophy at ScholarWorks, Georgia State University. http://scholarworks.gsu.edu/philosophy_theses/150 (accessed Mar 20, 2016). 
'Headspace', (2016) https://www.headspace.com/faqs/category/our-approach-to-meditationand-mindfulness (Accessed April 5).

Ihde, Don (1990) Technology and the Lifeworld: From Garden to Earth. Indiana: Indiana University Press.

Ingold, Tim (2010) 'The taxtility of making'. Cambridge Journal of Economics. 34: 91-102. Latour, B. (1993). We Have Never Been Modern. Trans. C. Porter. Cambridge, MA: Harvard University Press

Lindman, Pia (2006) 'The New York Times, Art and Affect: Re-enactments in grey-scale' in Graham Coulter-Smith and Maurice Owen (eds) Art in the Age of Terrorism. New York:

Paul Holberton

Liu, Bing (2015) Sentiment Analysis: Mining Opinions, Sentiments, and Emotions.

Cambridge: Cambridge University Press.

Lopatovska, Irene and Arapakis Ioannis (2011) 'Theories, Methods and Current Research on Emotions in Library and Information Science, Information Retrieval and Human-Computer Interaction', Information Processing and Management 47(4), July: 575-92.

Lugmayr, Artur, Dorsch, Tillmann, Humanes, Pablo Roman (2009) 'Emotional Ambient Media', in Handbook of Research on Synthetic Emotions and Sociable Robotics: New Applications in Affective Computing and Artificial Intelligence, pp. 443-459, by Jordi Vallverdy and David Casacuberta. Harshey and London: Information Science Reference. Myers, David G. (2005) Exploring psychology. New York: Worth Books.

O'Sullivan, Dan and Igoe, Tom (2004) Physical Computing: Sensing and Controlling the Physical World with Computers. Boston: Thomson Course Technology PTR.

Picard, Rosalind W. (1995) ‘Affective Computing' in M.I.T Media Laboratory Perceptual Computing Section Technical Report No. 321, Massachusetts Institute of Technology 
Picard, Rosalind W. (2007) 'Toward Machines with Emotional Intelligence', in The Science of Emotional Intelligence: Knowns and Unknowns, Matthews, G., Zeidner, M., \& Roberts, R. D. (Eds.) Oxford, UK: Oxford University Press, Pre-Print PDE at $<$ http://affect.media.mit.edu/pdfs/07.picard-EI-chapter.pdf> (accessed on 30 Mar 2016). Pickering, Andrew (2008) 'New ontologies', pp. 5 in Andrew Pickering and Keith Guzik (eds) The Mangle in Practice: Science, Society, and Becoming, Durham: Duke University Press.

Pickering, Andrew (2013) 'Being in an Environment: A Performative Perspective' Natures Sciences Sociétés 21 (1): 77-83.

Rosenberg, Terry E. (2013) 'Intermingled bodies, Distributed Agency in an Expanded Appreciation of Making'. FORMakademisk 6 (2): 1-18.

Rosenberger, Robert and Verbeek, Peter-Paul (eds) 2015. Postphenomenological Investigations: Essays on Human-Technology Relations. London: Lexington Books. Ryan, Susan Elizabeth (2014) Garments of Paradise: Wearable Discourse in the Digital Age. Massachusetts: MIT Press.

Turkle, Sherry (2011) Along Together: Why We Expect More from Technology and Less from Each Other. New York: Basic Books.

Verbeek, Peter-Paul(2015) 'Beyond interactions: a short introduction to mediation theory', Interactions, May-June 2015: 26-31.

Walker, Kevin, and Fass, John (2015) 'De-computation: programming the world through design', Nordes 2015: Design Ecologies. Nordic Design Research.

Wilkinson-Weber, Clare M. and DeNicola, Alicia Ory (eds) (2016) Critical Craft: Technology, Globalization, and Capitalism. London:Bloomsbury Publishing. Williams, Michele A., Roseway, Asta., O'Dowd, Chris, Czerwinski, Mary and Morris M. Ringel (2015) 'SWARM: An Actuated Wearable for Mediating Affect' in Proceedings of the 
Ninth International Conference on Tangible, Embedded, and Embodied Interaction, pp. 290300. ACM .

Złotowski, Jakub, Proudfoot, Diane, Yogeeswaran, Kumar and Bartneck, Christoph (2015)

'Anthropomorphism: Opportunities and Challenges in Human-Robot Interaction'.

International Journal of Social Robotics 7 (3): 347-60. 